\title{
Herzfrequenzmessung zur Bestimmung der Trainingsintensität in Lungensportgruppen ${ }^{1}$
}

\author{
Heart Rate Measurement for Determination of Training Intensity \\ in Outpatient Pulmonary Sport Groups
}

Autoren

Institute
H. J. Baumann' , S. Kluge², H. Klose', A. Hellweger ${ }^{3}$, K. M. Braumann³ , A. Meyer ${ }^{4}$

Zentrum für Innere Medizin, II. Medizinische Klinik, Sektion Pneumologie, Universitätsklinikum Hamburg-Eppendorf (Direktor: Prof. Dr. med. C. Bokemeyer)

Zentrum für Anästhesiologie und Intensivmedizin, Klinik für Intensivmedizin, Universitätsklinikum Hamburg-Eppendorf

3 Fachbereich Sportwissenschaft, Institut für Sport- und Bewegungsmedizin, Universität Hamburg

${ }^{4}$ Kliniken Mariahilf GmbH, Klinik für Pneumologie, Mönchengladbach eingereicht 19.7.2008

akzeptiert nach Revision 26.9.2008

Bibliografie

DOI $10.1055 / \mathrm{s}-2008-1038288$

Online-Publikation: 24.11.2008

Pneumologie 2009; 63: 72-77

(c) Georg Thieme Verlag KC

Stuttgart · New York

ISSN 0934-8387

\section{Korrespondenzadresse}

\section{Dr. Hans Jörg Baumann}

Sektion Pneumologie, II. Medizinische Klinik, Universitäts-

klinikum Hamburg-Eppendorf

Martinistr. 52

20246 Hamburg

hbaumann@uke.uni-hamburg.de

\section{Zusammenfassung \\ $\nabla$}

Hintergrund: Körperliches Training ist etablierter Bestandteil der Behandlung von Patienten mit Asthma und COPD. Zur Trainingssteuerung wird neben der spiroergometrisch ermittelten maximalen Sauerstoffaufnahme $\left(\mathrm{VO}_{2} \mathrm{max}\right)$ die Herzfrequenz genutzt. Es wird eine Trainingsintensität von $60-80 \%$ der $\mathrm{VO}_{2}$ max empfohlen. $\mathrm{Ob}$ diese Vorgabe in hinsichtlich der Teilnehmerstruktur heterogenen Lungensportgruppen erreicht werden kann, ist unklar.

Patienten und Methode: 61 Erwachsene $(m=15$, $w=46$, Alter: $63 \pm 9$ Jahre) mit Asthma bronchiale $(n=50)$ oder COPD $(n=11)$ trainierten in ambulanten Lungensportgruppen. Für jeden Patienten wurde ein Trainingsbereich von $60-80 \%$ der errechneten $\mathrm{VO}_{2}$ max entsprechend einer Herzfrequenz zwischen 97-137/min ermittelt. Die Pulsfrequenz wurde über jeweils $60 \mathrm{~min}$. elektronisch aufgezeichnet.

Ergebnisse: Alle Patienten bis auf einen Asthmatiker erreichten während der Ausdauerphase den Herzfrequenzzielbereich. Es wurden $81 \pm 9 \%$ des angestrebten Trainingsziels von geschätzt $80 \%$ VOmax erreicht (Asthmatiker: 79 $\pm 9 \%$, COPD-Patienten: $88 \pm 9 \%, \mathrm{p}<0,005)$. Unterschiede bei der erreichten Trainingsintensität fanden sich hinsichtlich Krankheitsschwere und Alter nicht.

Schlussfolgerung: In Lungensportgruppen mit heterogener Teilnehmerstruktur ist ein Training im Herzfrequenz-Zielbereich möglich. COPD-Patienten erreichten ein signifikant höheres, relatives Belastungsniveau. Die Pulsfrequenz eignet sich als einfach zu erhebender Parameter zur Trainingssteuerung.

\section{Abstract \\ $\nabla$}

Background: Exercise training is an integral component of the management of patients with asthma and COPD. A training intensity of $60-80 \%$ of maximum oxygen uptake $\left(\mathrm{VO}_{2} \max \right)$ has been recommended. Instead of ergospirometry measurement of heart rate can be used for training guidance. It is unknown whether the given target can be reached in outpatient training groups with substantial heterogeneity concering diagnosis, disease severity, and age.

Methods and patients: 61 adult patients $(\mathrm{m}=15$, $w=46 ; 63 \pm 9$ years $)$ with asthma $(n=50)$ or COPD $(n=11)$ exercised in groups of ambulant lung sports. An individualized target heart rate was calculated corresponding to an intensity of $60 \%$ to $80 \%$ of calculated $\mathrm{VO}_{2}$ max which was equivalent to a heart rate from 97 to 137 beats per minute in the population studied. Heart reate, as a measure of the intensity of charge, was measured continuously with pulse frequency meters.

Results: All but one asthma patient exercised in the targeted heart rate range. Patients reached $81 \pm 9 \%$ of the training goal of calculated $80 \%$ $\mathrm{VO}_{2} \max$ (asthma patients: $79 \pm 9 \%$ vs. COPD patients: $88 \pm 9 \%, \mathrm{p}<0,005)$ No significant differences regarding training intensities were observed in relation to severity of illness or age.

Conclusions: In outpatient lung sport groups of considerable heterogeneity regarding diagnosis, severity of illness and age, participants reach their individualized heart rate target range. COPD patients were training at a significantly higher relative training intensity. Heart rate is an easy to use parameter for training guidance.

\footnotetext{
${ }^{1}$ Die Ergebnisse dieser Studie basieren auf der Promotionsarbeit von Dr. A. Hellweger.
} 


\section{Einleitung \\ $\nabla$}

Körperliches Training ist fester Bestandteil der Rehabilitation von Patienten mit Asthma bronchiale oder COPD $[8,16,19,22,28,31]$. Dieses Training wird in der Regel im Rahmen einer stationären Rehabilitation in spezialisierten Kliniken durchgeführt. Die während der meist dreiwöchigen Rehabilitation erreichte Verbesserung der körperlichen Leistungsfähigkeit bleibt jedoch langfristig nur selten erhalten [4,22].

In den letzten 15 Jahren hat sich eine zunehmende Anzahl von ambulanten Lungensportgruppen in Deutschland etabliert, in denen Patienten mit obstruktiven Atemwegserkrankungen ihre körperliche Leistungsfähigkeit langfristig erhalten oder verbessern können [2]. Der Vorteil des wohnortnahen Angebots der Lungensportgruppen ist jedoch verknüpft mit dem Nachteil, dass in der verhältnismäßig geringen Anzahl bestehender Lungensportgruppen eine Differenzierung nach Diagnose, Alter, Leistungsfähigkeit und Schweregrad der Erkrankung nur begrenzt möglich ist. Ob die in den wegweisenden Studien erarbeiteten Zielvorgaben hinsichtlich der Trainingsintensität in der Realität der ambulanten Lungensportgruppen umgesetzt werden können, ist bislang nicht gezeigt worden [13].

Nicht abschließend geklärt ist, welches Trainingsziel beim körperlichen Training von Patienten mit Atemwegserkrankungen zu optimalen Resultaten führt [19]. In der Literatur wird eine Steuerung der Trainingsintensität mithilfe von vor Trainingsbeginn erhobenen Parametern der kardiopulmonalen Leistungsfähigkeit empfohlen $[1,15,19,32]$. Verwendet wird dazu in der Regel die Spiroergometrie zur Bestimmung der maximalen Sauerstoffaufnahme bzw. der Laktatschwelle. Während der Einsatz der Spiroergometrie zur Trainingssteuerung im Rahmen wissenschaftlicher Fragestellungen als Standard anzusehen ist, kann diese verhältnismäßig aufwändige Untersuchung im Breitensport nicht im eigentlich wünschenswerten Umfang eingesetzt werden. Einfacher zu erhebende Parameter für die Trainingssteuerung sind die Herzfrequenz sowie der subjektiv empfundene Dyspnoegrad [1,12,14,15].

Ziel der vorliegenden Untersuchung war es nun zu prüfen, ob in hinsichtlich der Teilnehmercharakteristika heterogenen Lungensportgruppen ein körperliches Training in der Art durchführbar war, dass der einzelne Teilnehmer im individuell vorgesehenen Herzfrequenzbereich belastet wurde.

\section{Methode \\ $\nabla$}

\section{Patienten}

Es wurden Patienten aus 7 der zum Studienzeitpunkt existierenden 23 Lungensportgruppen aus dem Großraum Hamburg untersucht. Die Auswahl der Gruppen erfolgte aus rein praktischen Erwägungen, weil diese für den Untersucher (A.H.) am besten erreichbar waren. Innerhalb der Lungensportgruppen wurden nicht alle Teilnehmer untersucht. Die Auswahl der Patienten erfolgte nach folgendem Schema: Für die Untersuchung standen 10 Pulsfrequenzmessgeräte zur Verfügung, die in den Gruppen an alle Teilnehmer verteilt wurden. War die Anzahl der Teilnehmer größer als 10, so wurden die ersten 10 Teilnehmer ausgewählt, die zuerst am Trainingsort eintrafen.

61 Patienten ( $m=15, w=46$, mittleres Alter: $63 \pm 9$ (31 - 84) Jahre), davon 50 mit klinisch stabilem Asthma bronchiale (leichtgradig: $n=21$; mittelgradig: $n=16$; schwergradig: $n=13$, Einteilung nach [7]) und 11 mit einer COPD (Schweregrad I: $n=5$;
Tab.1 Demografik und Medikation der Asthmapatienten.

\begin{tabular}{ll} 
Anzahl & 50 \\
\hline $\mathrm{m} / \mathrm{w}$ & $12 / 38$ \\
\hline Alter [Jahre] & $62 \pm 9(31-79)$ \\
$\mathrm{FEV}_{1}[\mathrm{l}]$ & $2,0 \pm 0,7(0,7-3,9)$ \\
\hline $\mathrm{FEV}_{1} \%$ Soll [\%] & $76 \pm 27(21-124)$ \\
$\mathrm{VK}$ Soll [\%] & $96 \pm 7(68-112)$ \\
\hline $\mathrm{FEV}_{1} / \mathrm{VK}[\%]$ & $74 \pm 23(46-91)$ \\
\hline inhalative Steroide & $46 / 50$ \\
\hline systemische Steroide & $16 / 50$ \\
\hline Theophyllin & $19 / 50$ \\
\hline langwirksame Betamimetika & $37 / 50$
\end{tabular}

Mittelwerte \pm Standardabweichung (Range); $m=$ männlich; $w$ = weiblich; $\mathrm{FEV}_{1}=$ forcierte expiratorische Einsekundenkapazität; $\mathrm{FEV}_{1} \%$ Soll = Einsekundenkapazität in Prozent vom Soll; VK\% Soll = Vitalkapazität in Prozent vom Soll.

Tab.2 Demografik und Medikation der COPD-Patienten.

\begin{tabular}{|c|c|}
\hline $\mathrm{m} / \mathrm{w}$ & $3 / 8$ \\
\hline Alter [Jahre] & $70 \pm 7,8(56-84)$ \\
\hline $\mathrm{FEV}_{1}[\mathrm{I}]$ & $1,8 \pm 0,5(0,9-2,3)$ \\
\hline $\mathrm{FEV}_{1} \%$ Soll [\%] & $79 \pm 18(58-121)$ \\
\hline VK\% Soll [\%] & $113 \pm 12(70-136)$ \\
\hline $\mathrm{FEV}_{1} / \mathrm{VK}[\%]$ & $67 \pm 3(43-70)$ \\
\hline inhalative Steroide & $7 / 11$ \\
\hline systemische Steroide & $3 / 11$ \\
\hline Theophyllin & $3 / 11$ \\
\hline langwirksame Betamimetika & $6 / 11$ \\
\hline \multicolumn{2}{|c|}{$\begin{array}{l}\text { Mittelwerte } \pm \text { Standardweichung (Range); } m=\text { männlich; } w=\text { weiblich; } \\
\mathrm{FEV}_{1}=\text { = forcierte expiratorische Einsekundenkapazität; } \mathrm{FEV}_{1} \% \text { Soll = Ein- } \\
\text { sekundenkapazität in Prozent vom Soll; VK\% Soll = Vitalkapazität in Prozent } \\
\text { vom Soll. }\end{array}$} \\
\hline
\end{tabular}

Schweregrad II: n=6, Einteilung nach [28]), nahmen einmal wöchentlich an einem ambulanten Trainingsprogramm teil. - Tab. 1 zeigt die Charakteristika der Patienten mit Asthma bronchiale, Tab. 2 die der Patienten mit COPD. Alle Patienten wurden ausschließlich von niedergelassenen Pneumologen den Lungensportgruppen entsprechend den Empfehlungen der Deutschen Atemwegsliga zum körperlichen Training zugewiesen [32]. Die Diagnose der Patienten wurde durch die jeweils zuständigen Pneumologen gestellt. Diagnostik und Therapie der Erkrankungen erfolgten nach den aktuellen Empfehlungen der Fachgesellschaften $[7,28]$. Eine zusätzliche kardiopulmonale Diagnostik der Patienten im Rahmen der Studie fand nicht statt. In Diskrepanz zu den geltenden Empfehlungen wurde ein AsthmaPatient nicht leitliniengerecht ohne inhalative Steroide behandelt. Sowohl einige der leichtgradig erkrankten Asthmatiker als auch der leichtgradigen COPD-Patienten erhielten, anders als in den Leitlinien vorgesehen, langwirksame Bronchodilatatoren. Die Angaben zur Gabe systemischer Steroide beziehen sich auf den Zeitraum des vor dem Untersuchungszeitpunkt liegenden halben Jahres. Alle Patienten trainierten seit mindestens einem Jahr in den Lungensportgruppen.

\section{Beurteilung der Belastungsintensität}

In den untersuchten Lungensportgruppen wurde gemäß den geltenden Empfehlungen ein Training mit submaximaler Intensität angestrebt [12,32]. Die Belastungsintensität wurde indirekt anhand der beim Patienten gemessenen Herzfrequenz in Relation zu den errechneten Sollwerten gemäß Tab. 3 beurteilt. - Tab. 3 zeigt den Zusammenhang zwischen maximaler Sauer- 
Tab.3 Pulsfrequenz-Richtwerte zur Bemessung der Belastungsintensität in der jeweiligen Altersgruppe (aus [30]).

\begin{tabular}{llll}
$\begin{array}{l}\text { Alter } \\
\text { (Jahre) }\end{array}$ & $\begin{array}{l}\mathbf{6 0} \% \\
\mathbf{V O}_{2} \mathbf{m a x}\end{array}$ & $\begin{array}{l}\mathbf{7 0} \% \\
\mathbf{V O}_{\mathbf{2}} \mathbf{m a x}\end{array}$ & $\begin{array}{l}\mathbf{8 0} \% \\
\mathbf{V O}_{\mathbf{2}} \mathbf{m a x}\end{array}$ \\
$30-35$ & $130 / \mathrm{min}$ & $150 / \mathrm{min}$ & $170 / \mathrm{min}$ \\
\hline $36-40$ & $125 / \mathrm{min}$ & $145 / \mathrm{min}$ & $165 / \mathrm{min}$ \\
\hline $41-45$ & $120 / \mathrm{min}$ & $140 / \mathrm{min}$ & $160 / \mathrm{min}$ \\
\hline $45-50$ & $115 / \mathrm{min}$ & $135 / \mathrm{min}$ & $155 / \mathrm{min}$ \\
\hline $51-55$ & $110 / \mathrm{min}$ & $130 / \mathrm{min}$ & $150 / \mathrm{min}$ \\
\hline $56-60$ & $105 / \mathrm{min}$ & $125 / \mathrm{min}$ & $145 / \mathrm{min}$ \\
\hline $61-65$ & $100 / \mathrm{min}$ & $120 / \mathrm{min}$ & $140 / \mathrm{min}$ \\
\hline $66-70$ & $95 / \mathrm{min}$ & $115 / \mathrm{min}$ & $135 / \mathrm{min}$ \\
\hline $71-75$ & $90 / \mathrm{min}$ & $110 / \mathrm{min}$ & $130 / \mathrm{min}$ \\
\hline Faustregel: & $160 / \mathrm{min}-$ Alter & $180 / \mathrm{min}-$ Alter & $200 / \mathrm{min}-$ Alter \\
\hline
\end{tabular}

stoffaufnahme, Alter und Herzfrequenz, der für die Beurteilung der Belastungsintensität zugrunde gelegt wurde (aus [30]). Eine Spiroergometrie vor Beginn der Teilnahme am Lungensport war keine Voraussetzung und erfolgte auch nur bei einzelnen Patienten und zudem in größerem zeitlichen Abstand zum Zeitpunkt der Studie.

\section{Trainingsprogramm}

Das Training fand einmal wöchentlich bei einer Gruppenstärke von 10-20 Patienten statt. Die Lokalität stellten die Sportstätten bzw. Turnhallen der beteiligten Sportvereine dar. Die Trainingszeit betrug 60 Minuten. Angeleitet wurden die Patienten durch speziell ausgebildete Übungsleiter. Vor und nach jeder Trainingseinheit wurde der Peak flow gemessen und die Befindlichkeit auf einer Skala von $1-3$ abgefragt $(1=$ schlecht, $3=$ gut $)$. Voraussetzung für eine Teilnahme am Training waren Peak flowWerte, die im Bereich von $80-100 \%$ des persönlichen Bestwertes lagen, und eine Befindlichkeit von mindestens 2. Alle Patienten wurden angewiesen, mindestens $30 \mathrm{~min}$. vor Beginn der Trainingseinheit ihren kurzwirksamen Bronchodilatator zu inhalieren.

Das Trainingsprogramm bestand aus 3, auf das Krankheitsbild ausgerichteten Teilen. Im 1. Teil erfolgte eine ca. 15 Minuten dauernde Erwärmung. Hier wurden schnelles Gehen sowie Gymnastik und Stretching zur Lockerung und Kräftigung von Schultergürtel und Rückenmuskulatur durchgeführt. Koordinations-, Kraft- und speziell Ausdauerschulung, zum Teil als Zirkeltraining, sowie Atemgymnastik zur Kräftigung von Zwerchfell und Atemhilfsmuskulatur waren Bestandteile des ca. 30 Minuten dauernden 2. Teils. Hier wurde die höchste Belastungsintensität angestrebt. Die Intensität wurde stichpunktartig mittels selbstständiger Pulskontrolle durch den Patienten überprüft. Regelmäßig erfolgte auch das Einüben von Atemtechniken wie Lippenbremse unter Belastung und atemerleichternden Stellungen. Der 3. Teil mit einer Dauer von 15 Minuten bestand aus Dehnlagen nach Scharschuch/Haase und Entspannungsübungen, meist als progressive Muskelrelaxation nach Jakobson.

\section{Organisation}

Träger der ambulanten Rehabilitation durch Sport ist in Hamburg der Behinderten- und Rehabilitations-Sportverband Hamburg e.V. Die Gruppen sind Bestandteil der lokalen Sportvereine, deren Infrastruktur für den Aufbau und die Organisation der Sportgruppen genutzt werden konnte. Die Teilnehmer am ambulanten Rehabilitationssport werden Mitglieder im jeweiligen
Sportverein und tragen den Vereinsbeitrag von 7-10EUR pro Monat selbst. Die Krankenkassen unterstützen den Rehabilitationssport mit zurzeit etwa 3,5EUR pro Teilnehmer und Stunde.

\section{Datenerhebung}

Bei allen Patienten wurde an 3 unterschiedlichen Trainingsterminen die Pulsfrequenz kontinuierlich apparativ aufgezeichnet und später mit einem Computerprogramm ausgewertet. Sowohl Patienten als auch Übungsleiter waren während der Übungseinheit nicht über die so gemessene Herzfrequenz informiert. Verwendet wurden Accurex plus Pulsuhren (Firma Polar Herzfrequenz-Messgeräte/Finnland), die die Herzfrequenz alle 5 Sekunden über der Brustwand ableiten und intern abspeichern. Im 5 Sekunden-Messintervall lag der Fehler dieser Pulsuhren bei unter $1 \%[15]$.

\section{Statistik}

Für die statistische Auswertung wurde das Statistikprogramm „XXL-Stats“ in Microsoft-Excel benutzt. Die Ergebnisse wurden als Mittelwerte mit Standardabweichungen angegeben. Unterschiede zwischen den Gruppen wurden mittels unverbundenem t-Test ermittelt. Ein $\mathrm{p}<0,05$ wurde dabei als Signifikanzniveau festgelegt. Unterschiede zwischen den Trainingsphasen (Erwärmung vs. Ausdauer vs. Erholung) wurden mittels One-way-ANOVA für Messwertwiederholungen untersucht, auch hier galt als Signifikanzniveau $\mathrm{p}<0,05$.

\section{Ergebnisse \\ $\nabla$}

Für jeden einzelnen Patienten wurde entsprechend der o.g. Formel $\mathrm{HF}_{\max }=200$-Lebensalter die für den Rehabilitationssport empfohlene mittlere maximale Pulsfrequenz errechnet. Für die Gruppe aller Patienten ergab sich bei einem durchschnittlichen Alter von 63 Jahren eine angestrebte Pulsfrequenz von 137/min. Der effektivste Trainingsbereich liegt dann (bei dieser nomogrammatisch ermittelten Intensität, entsprechend 0 Tab.3) zwischen $97 / \mathrm{min}$ (formal entsprechend $60 \% \mathrm{VO}_{2} \max$ ) und $137 /$ min (formal entsprechend $80 \% \mathrm{VO}_{2}$ max). Die durchschnittliche Pulsfrequenz lag in der Gesamtgruppe bei $104 \pm 12 / \mathrm{min}$ während des gesamten Trainings, was ca. $64 \%$ der erwarteten $\mathrm{VO}_{2}$ max entspricht. Während der Aufwärmphase wurde eine mittlere Pulsfrequenz von $100 \pm 11 / \mathrm{min}\left(62 \% \mathrm{VO}_{2} \mathrm{max}\right)$, in der Ausdauerphase $110 \pm 14 / \mathrm{min}\left(67 \% \mathrm{VO}_{2} \max \right)$ und in der Entspannungsphase $97 \pm 13 / \min \left(60 \% \mathrm{VO}_{2} \max \right)$ gemessen. Es fanden sich signifikante Unterschiede hinsichtlich der Pulsfrequenz zwischen Aufwärm- und Ausdauerphase sowie Ausdauer- und Erholungsphase $(\mathrm{p}<0,001)$. $\odot$ Abb. 1 zeigt die von den Asthma bronchiale- bzw. COPD-Patienten während der Trainingsphasen erreichten Pulsfrequenzen in Relation zum angestrebten Trainingsbereich. Lediglich ein Patient mit Asthma erreichte den Herzfrequenzzielbereich während der Ausdauerphase nicht (59\% der kalkulierten $\mathrm{VO}_{2} \mathrm{max}$ ). Es konnten keine signifikanten Korrelationen zwischen Schweregrad der Erkrankung, Alter, Geschlecht und erreichter Trainingsintensität bei den Patienten mit Asthma bronchiale festgestellt werden (leichtgradig: 79\%, mittelgradig: $81 \%$, schwergradig: $78 \%$, männlich: $79 \%$, weiblich: $79 \%$ des angestrebten Zielwertes von $80 \% \mathrm{VO}_{2} \mathrm{max}$, sämtliche Korrelationen $r<0,5)$. Die COPD-Patienten erreichten ein signifikant höheres, relatives Belastungsniveau: $88 \pm 9 \%$ des angestrebten Zielwertes von $80 \% \mathrm{VO}_{2}$ max im Vergleich zu $79 \pm 9 \%$ bei den Asthma-Patienten $(\mathrm{p}<0,005)$. 


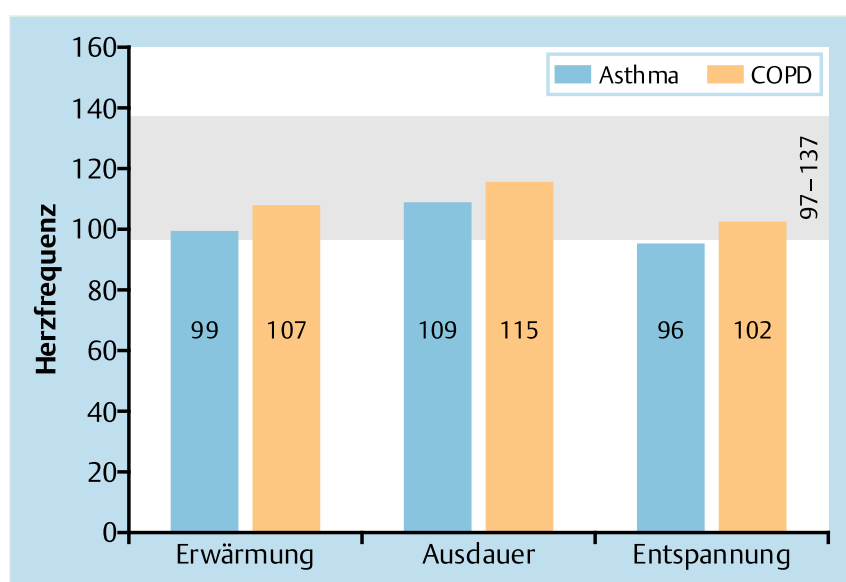

Abb. 1 Durchschnittlliche Herzfrequenzen, die von den Asthma- bzw. COPD-Patienten während der Trainingsphasen in Relation zum Herz-Kreislauf-effektiven Trainingsbereich erreicht wurden.

Die Peak flow-Werte und die subjektive Befindlichkeit wurden durch die Trainingseinheiten nicht beeinflusst.

\section{Diskussion}

Die vorliegende Arbeit belegt mittels Untersuchung der durchschnittlichen Herzfrequenz von Asthma- bzw. COPD-Patienten während des körperlichen Trainings, dass in hinsichtlich Erkrankungsschwere, Alter und körperlicher Leistungsfähigkeit der Teilnehmer heterogenen Lungensportgruppen ein an die individuell erforderliche Intensität angepasstes Training durchführbar ist.

Das Management einer chronischen Krankheit erfordert nicht nur eine optimale Diagnostik und Therapie, sondern hat auch zum Ziel, die Folgen der Chronizität der Erkrankung zu mindern. Neben einer an den Schweregrad angepassten, medikamentösen Therapie ist die Sport- und Bewegungstherapie fester Bestandteil im Behandlungskonzept des Asthma bronchiale bzw. der COPD $[7,16,17,28]$. Empfohlen wird ein körperliches Training vor allem für Patienten mit mittelschwerem bis schwerem Krankheitsbild, aber auch für Patienten mit leichtgradiger Einschränkung der Lungenfunktion und ausgeprägtem Mangel an körperlicher Leistungsfähigkeit [10,32]. In Lungensportgruppen haben Patienten mit chronisch-obstruktiven Atemwegserkrankungen die Möglichkeit, ambulant und langfristig ihre körperliche Leistungsfähigkeit zu erhalten oder zu verbessern [17,32]. Im Rehabilitationssport wird ein Training im submaximalen Bereich angestrebt $[1,12]$. Die körperliche Belastung sollte deutlich über der durchschnittlichen Alltagsbelastung liegen, die etwa $30 \%$ der maximalen Sauerstoffaufnahme $\left(\mathrm{VO}_{2} \mathrm{max}\right)$ entspricht [29]. Bei $80 \%$ der $\mathrm{VO}_{2}$ max ist bei Gesunden in der Regel die anaerobe Schwelle erreicht $[15,29,30]$. Der effektivste Belastungsbereich liegt im aeroben Bereich, also bei $60-80 \%$ der $\mathrm{VO}_{2} \max$ [1]. Bei Patienten mit obstruktiven Atemwegserkrankungen ist der limitierende Faktor bei körperlicher Belastung in der Regel die Atemnot [16,21,31]. Die Regeln der Leistungsphysiologie lassen sich im aeroben Ausdauertraining auch bei diesem Kollektiv anwenden $[20,25,32]$. Idealerweise wird der individuell optimale Trainingsbereich durch einen Facharzt vor Beginn des Trainings festgelegt, am besten durch spiroergometrische Bestimmung der $\mathrm{VO}_{2}$ max.
Die Pulsfrequenz korreliert bei Gesunden im aeroben Trainingsbereich gut mit der Sauerstoffaufnahme [29,30]. $\bullet$ Tab. 3 zeigt den Zusammenhang zwischen maximaler Sauerstoffaufnahme, Alter und Herzfrequenz (aus [30]). Diese Relation findet sich auch bei Patienten mit COPD [25]. Es gibt jedoch Faktoren, die den linearen Zusammenhang zwischen Herzfrequenz und $\mathrm{VO}_{2}$ beeinflussen können [6]: So kann die bei COPD-Patienten unter Belastung oft zu beobachtende dynamische Überblähung die Herzfrequenz über die lineare Beziehung zur $\mathrm{VO}_{2}$ hinaus steigern. Zudem erreichen Patienten mit chronisch-obstruktiver Ventilationsstörung bei niedrigeren $\mathrm{VO}_{2}$-Werten die anaerobe Schwelle [9]. Diese Beobachtung führte zu der Empfehlung, Trainingsprogramme für COPD- bzw. Asthma-Patienten ausschließlich an Spiroergometrie-Ergebnissen auszurichten [6]. Vallet u. Mitarb. konnten zeigen, dass eine individualisierte, auf die spiroergometrisch ermittelte anaerobe Schwelle bezogene Festlegung des Herzfrequenz-Zielbereichs bessere Trainingseffekte ergab als ein standardisiertes Vorgehen [27].

In der vorliegenden Arbeit wurde die Spiroergometrie nicht zur Trainingssteuerung verwendet. Ähnlich wie auch im Breitensport der Gesunden nicht nach spiroergometrisch ermittelten Vorgaben trainiert wird, erscheint in der breiten Anwendung des Lungensports die Nutzung der Spiroergometrie zwar wünschenswert, jedoch vor dem Hintergrund begrenzter Ressourcen nicht regelhaft einsetzbar. Wie weit man von der Forderung nach einer Spiroergometrie-basierten Trainingssteuerung entfernt ist, zeigt die Beobachtung, dass auch in dem von uns untersuchten Kollektiv nur vereinzelt eine Belastungsuntersuchung vor Beginn der Teilnahme am ambulanten Lungensport vorlag. Da in den Lungensportgruppen über Jahre hinweg trainiert wird, würde sich die Frage stellen, in welchen Zeiträumen die Spiroergometrie wiederholt werden müsste, um den optimalen Trainingsbereich auch im zeitlichen Verlauf korrekt angeben zu können.

Zudem ist die als Goldstandard anzusehende Spiroergometrie auch nicht immer in der Lage, einen exakten Herzfrequenz-Bereich der idealen Trainingsintensität festzulegen, z.B. wenn die anaerobe Schwelle nicht bestimmt werden kann oder unterschiedliche Methoden unterschiedliche Zielbereiche für die Trainingsherzfrequenz anzeigen [34].

Weil die Herzfrequenz einen in der Routine leicht zu bestimmenden Parameter darstellt, empfehlen die Leitlinien zur pulmonalen Rehabilitation trotz der genannten Einschränkungen neben der Verwendung der subjektiv empfundenen Dyspnoe insbesondere die Herzfrequenz zur Steuerung der Belastungsintensität $[5,14,19,32]$. Es wird dabei ein individuell festgelegter Bereich von 60 - 80\% der Herzfrequenz, die während eines initial durchgeführten Tests der maximalen Leistungsfähigkeit erreicht wurde, empfohlen. Die für den Rehabilitationssport empfohlene maximale Pulsfrequenz (200/min - Lebensalter) sollte in der Regel nicht überschritten werden $[10,16]$.

Das von uns untersuchte Kollektiv spiegelt die bezüglich Diagnose, Schweregrad der Erkrankung, Alter und Geschlecht sehr heterogene Zusammensetzung in den Lungensportgruppen wider. Dass alle Teilnehmer von dem angebotenen Training profitieren und tatsächlich im Herz-Kreislauf-effektiven Bereich arbeiten, stellt hohe Anforderungen an den verantwortlichen Übungsleiter. Die Übungseinheiten müssen so ausgewählt werden, dass eine Teilnahme mit unterschiedlichem Leistungsniveau möglich ist. Vor diesem Hintergrund ist die Forderung nach einer speziellen Übungsleiterausbildung für die Gruppenleiter im Lungensport offensichtlich sinnvoll. 
Die Steuerung der Trainingsintensität wird vom Übungsleiter aufgrund seiner Erfahrung, der Kenntnis der individuellen Voraussetzungen der Teilnehmer und der optischen Einschätzung während des Trainings vorgenommen. Diese subjektive Einschätzung sollte durch punktuelle Pulsfrequenzmessungen überprüft werden, die die Teilnehmer selbst vornehmen. In der Praxis der Lungensportgruppen zeigte sich, dass die anfangs häufig durchgeführten Herzfrequenzkontrollen im Verlauf zunehmend überflüssig wurden, da sich die Patienten auch ohne Pulsfrequenzkontrolle hinsichtlich der Trainingsintensität überwiegend gut selbst einschätzen konnten.

In der von uns durchgeführten Untersuchung wurde die individuelle Trainingsintensität mittels elektronischer Pulsuhren erfasst. Diese finden zur Trainingssteuerung im Leistungssport eine breite Anwendung, da sie ohne großen Aufwand anwendbar sind. Es zeigte sich, dass nahezu alle Patienten unabhängig von Alter, Geschlecht und Ausprägung der Erkrankung im HerzKreislauf effektiven Bereich trainierten. Die in den wissenschaftlichen Arbeiten zum körperlichen Training bei Asthma- bzw. COPD-Patienten erarbeiten Vorgaben für ein effizientes Training konnten also im Alltag der Lungensportgruppen umgesetzt werden.

Im Vergleich zu den Patienten mit Asthma bronchiale lag die Pulsfrequenz bei den COPD-Patienten bereits in der Erwärmung höher. Während des Ausdauertrainings fand sich dann ein signifikanter Unterschied ( $\mathrm{p}<0,007)$. Bedingt ist dies wahrscheinlich durch einen ausgeprägteren Konditionsmangel im Kollektiv der COPD-Patienten. Der höhere Puls während der Erwärmungsphase bzw. während der Belastung könnten auch durch die begleitende Medikation (s. T Tab.2) verursacht worden sein. Einige Patienten mit COPD erhielten als Dauertherapie Theophyllin oder langwirksame $\beta_{2}$-Mimetika. Während ein Anstieg der Pulsfrequenz durch kurzwirksame $\beta_{2}$-Mimetika nur etwa $20-30 \mathrm{Mi}-$ nuten anhält, führt die Einnahme von retardiertem Theophyllin und langwirksamen $\beta_{2}$-Mimetika zu einer längerfristigen Erhöhung des Ruhepulses um etwa 10-15\% [24]. Der Anstieg der Pulsfrequenz unter körperlicher Belastung wird allerdings nicht beeinflusst $[23,26]$. In einer Untersuchung von Belastungsprofilen bei Patienten mit schwerer COPD konnte mittels mobiler Spiroergometrie gezeigt werden, dass bereits bei geringer Alltagsbelastung die Ventilation schnell über dem Atemgrenzwert liegt und die Pulsfrequenz diesem Verlauf exakt folgt [18].

Betablocker und Verapamil können durch ihren negativ chronotropen Effekt die Verwendbarkeit der Pulsfrequenz als Instrument zur Trainingssteuerung beeinflussen [33]. Ein COPD-Patient wurde mit Betablockern behandelt, 4 weitere mit Calciumantagonisten. Alle erreichten dennoch den angestrebten Herzfrequenzbereich. In der Gruppe der Asthma-Patienten wurde kein Patient mit derartigen Medikamenten behandelt. Es muss darauf hingewiesen werden, dass bei mit herzfrequenzmodulierenden Medikamenten behandelten Patienten neben der Pulsfrequenz auch andere Kriterien der Belastung (subjektive Einschätzung, Dyspnoe, ggf. spiroergometrische Daten) zur Trainingssteuerung hinzugezogen werden müssen.

Bei COPD-Patienten im fortgeschrittenen Krankheitsstadium konnte eine Einschränkung der kardialen autonomen Regulation nachgewiesen werden, erkennbar u.a. an einer verminderten Herzfrequenzvariabilität [3]. Diese Effekte könnten die Nutzung der Pulsfrequenz zur Trainingssteuerung einschränken. Die Daten wurden jedoch an erheblich schwerer erkrankten Patienten erhoben als den hier vorgestellten ( $\mathrm{FEV}_{1} 35$ vs. $79 \%$ des Solls). Zudem konnten Costes u. Mitarb. zeigen, dass die Sensitivität des kardialen Baroreflexes sich infolge körperlichen Trainings wieder bessern kann [11].

In der vorliegenden Arbeit wurden lediglich Patienten mit obstruktiven Ventilationsstörungen untersucht, die COPD-Patienten wiesen einen leichten bis mittleren Schweregrad auf. Ob sich die gefundenen Ergebnisse auch auf Patienten mit restriktiven Ventilationsstörungen bzw. Patienten mit schwergradiger COPD übertragen lassen, bleibt ungewiss.

Zusammenfassend lassen die in der vorliegenden Studie gemachten Beobachtungen 3 wichtige Schlussfolgerungen zu:

- Trotz eines hinsichtlich Diagnose, Krankheitsschwere und Alter heterogenen Teilnehmerkollektives ist in Lungensportgruppen ein Training im angestrebten Bereich für jedes Individuum möglich.

- Die untersuchten COPD-Patienten erreichten im Vergleich zu Patienten mit Asthma bronchiale ein signifikant höheres, relatives Belastungsniveau.

- Die kontinuierliche Pulsfrequenzmessung mit elektronischen Pulsuhren kann zur Trainingssteuerung in Lungensportgruppen eingesetzt werden, wobei jedoch Einschränkungen bei der Annahme einer linearen Beziehung zwischen Herzfrequenz und $\mathrm{VO}_{2}$ bei Patienten mit pulmonalen Erkrankungen berücksichtigt werden müssen.

Vor dem Hintergrund dieser Ergebnisse halten wir es für notwendig, den Aufbau von Lungensportgruppen weiter zu fördern, so dass möglichst viele Patienten mit chronisch obstruktiven Atemwegserkrankungen von einer kontinuierlich durchgeführten Sport- und Bewegungstherapie profitieren können.

\section{Anmerkung \\ $\nabla$}

Dr. Baumann war an der Auswertung der Daten und Erstellung des Manuskripts beteiligt, Dr. Kluge und Dr. Klose haben zur Interpretation der Daten und der Erstellung des Manuskripts beigetragen, Dr. Hellweger war an der Konzeption der Studie und der Erstellung des Manuskripts beteiligt und hat die Daten erhoben, Prof. Braumann war an der Konzeption der Studie beteiligt und hat bei der Interpretation der Daten mitgeholfen, Dr. Meyer hat die Studie konzipiert und war an der Interpretation der Daten sowie der Erstellung des Manuskriptes beteiligt.

\section{Literatur}

1 ACSM. American College of Sports Medicine Position Stand. The recommended quantity and quality of exercise for developing and maintaining cardiorespiratory and muscular fitness, and flexibility in healthy adults. Med Sci Sports Exerc 1998; 30: 975 - 991

2 AG Lungensport Hamburg e.V.. Available at: www.lungensport.de. Accessed 02.08. 2008

3 Bartels MN, Jelic S, Ngai P et al. High-frequency modulation of heart rate variability during exercise in patients with COPD. Chest 2003; 124: $863-869$

4 Bestall JC, Paul EA, Garrod R et al. Longitudinal trends in exercise capacity and health status after pulmonary rehabilitation in patients with COPD. Respir Med 2003; 97: 173-180

5 British Thoracic Society. Pulmonary rehabilitation. Thorax 2001; 56: $827-834$

6 Brolin SE, Cecins NM, Jenkins SC. Questioning the use of heart rate and dyspnea in the prescription of exercise in subjects with chronic obstructive pulmonary disease. J Cardiopulm Rehabil 2003; 23: 228 234

7 Buhl R, Berdel D, Criee CP et al. Leitlinie zur Diagnostik und Therapie von Patienten mit Asthma - Herausgegeben von der Deutschen Atemwegsliga und der Deutschen Gesellschaft fur Pneumologie und Beatmungsmedizin e.V. Pneumologie 2006; 60: 139-177 
8 Cambach W, Chadwick-Straver RV, Wagenaar RC et al. The effects of a community-based pulmonary rehabilitation programme on exercise tolerance and quality of life: a randomized controlled trial. Eur Respir J 1997; 10: $104-113$

9 Casaburi R, Patessio A, Ioli F et al. Reductions in exercise lactic acidosis and ventilation as a result of exercise training in patients with obstructive lung disease. Am Rev Respir Dis 1991; 143: 9- 18

10 Clark CJ. The role of physical training in asthma. In: Casaburi R, Petty $\mathrm{TL}$ (eds). Principles and practice of pulmonary rehabilitation. Philadelphia: Saunders, 1993

11 Costes F, Roche F, Pichot V et al. Influence of exercise training on cardiac baroreflex sensitivity in patients with COPD. Eur Respir J 2004; 23: $396-401$

12 Göhl O, Pleyer K, Biberger $G$ et al. Empfehlungen zur Planung und Durchführung des körperlichen Trainings im Lungensport. Pneumologie 2006; 60: 716 - 723

13 Griffiths TL, Burr ML, Campbell IA et al. Results at 1 year of outpatient multidisciplinary pulmonary rehabilitation: a randomised controlled trial. Lancet 2000; 355: $362-368$

14 Horowitz MB, Littenberg B, Mahler DA. Dyspnea ratings for prescribing exercise intensity in patients with COPD. Chest 1996; 109: 1169-1175

15 Janssen PG. Trainingssteuerung über Herzfrequenz- und Milchsäurebestimmungen. Balingen: Perimed-Spitta-Verlag, 1996

16 Lecheler J, Biberger A, Pfannebecker B. Asthma und Sported. Berchtesgarden: INA-Verlag, 2006

17 Meyer A, Wendt G, Taube K et al. [Ambulatory sports in asthma improves physical fitness and reduces asthma-induced hospital stay]. Pneumologie 1997; 51: 845 - 849

18 Müller C, Weißmeier F, Halle $M$ et al. Ventilatorische Belastung im ambulanten Lungensport - Falldarstellung. Phys Rehab Kur Med 2005; 15

19 Nici L, Donner C, Wouters E et al. American Thoracic Society/European Respiratory Society statement on pulmonary rehabilitation. Am J Respir Crit Care Med 2006; 173: 1390 - 1413

20 Pleyer K, Schmitz M. Sport und Bewegung in der Asthmatherapie. Pneumologie 1998; 52: $41-51$

21 Ram FS, Robinson SM, Black PN et al. Physical training for asthma. Cochrane Database Syst Rev 2005; CD001116

22 Ries AL, Kaplan RM, Myers $R$ et al. Maintenance after pulmonary rehabilitation in chronic lung disease: a randomized trial. Am J Respir Crit Care Med 2003; 167: 880-888
23 Saito S, Miyamoto K, Nishimura M et al. Effects of inhaled bronchodilators on pulmonary hemodynamics at rest and during exercise in patients with COPD. Chest 1999; 115: 376- 382

24 Salpeter SR, Ormiston TM, Salpeter EE. Cardiovascular effects of betaagonists in patients with asthma and COPD: a meta-analysis. Chest 2004; 125: 2309-2321

25 Simmons DN, Berry MJ, Hayes SI et al. The relationship between $\% \mathrm{HR}_{\text {peak }}$ and $\mathrm{VO}_{2 \text { peak }}$ in patients with chronic obstructive pulmonary disease. Med Sci Sports Exerc 2000; 32: 881-886

26 Tsukino M, Nishimura K, Ikeda A et al. Effects of theophylline and ipratropium bromide on exercise performance in patients with stable chronic obstructive pulmonary disease. Thorax 1998; 53: 269-273

27 Vallet G, Ahmaidi S, Serres I et al. Comparison of two training programmes in chronic airway limitation patients: standardized versus individualized protocols. Eur Respir J 1997; 10: 114-122

28 Vogelmeier C, Buhl R, Criee CP et al. Leitlinie der Deutschen Atemwegsliga und der Deutschen Gesellschaft fur Pneumologie und Beatmungsmedizin zur Diagnostik und Therapie von Patienten mit chronisch obstruktiver Bronchitis und Lungenemphysem (COPD). Pneumologie 2007; 61: e1 - 40

29 Wasserman K, Hansen J, Sue DY et al. Principles of exercise testing and interpretation. 4th ed. Philadelphia: Lippincott Williams \& Wilkins, 2005

30 Weineck J. Optimales Training. Leistungsphysiologische Trainingslehre. Balingen: Spitta Verlag, 1997

31 Worth H, Buhl R, Cegla U et al. Leitlinie der Deutschen Atemwegsliga und der Deutschen Gesellschaft für Pneumologie zur Diagnostik und Therapie von Patienten mit chronisch obstruktiver Bronchitis und Lungenemphysem (COPD). Pneumologie 2002; 56: 704-738

32 Worth $\mathrm{H}$, Meyer A, Folgering $\mathrm{H}$ et al. Empfehlungen der Deutschen Atemwegsliga zum Sport und körperlichen Training bei Patienten mit obstruktiven Atemwegserkrankungen. Pneumologie 2000; 54: $61-67$

33 Yamakado T, Oonishi N, Kondo S et al. Effects of diltiazem on cardiovascular responses during exercise in systemic hypertension and comparison with propranolol. Am J Cardiol 1983; 52: 1023-1027

34 Zacarias EC, Neder JA, Cendom SP et al. Heart rate at the estimated lactate threshold in patients with chronic obstructive pulmonary disease: effects on the target intensity for dynamic exercise training. J Cardiopulm Rehabil 2000; 20: 369-376 